\title{
Diagnóstico diferencial de las melanosis orofaciales
}

\section{Differential diagnosis of orofacial melanosis}

\section{Q1Eva López-Montoro ${ }_{\wedge}^{*}$ y Eduardo Chimenos-Küstner \\ Departamento de Odontoestomatología, Facultad de Medicina y Ciencias de la Salud, Universidad de Barcelona, Barcelona, España}

\section{Introducción}

En los tejidos tegumentarios (piel y mucosas) asientan con cierta frecuencia lesiones oscuras. A menudo se forman por la inducción de los melanocitos a producir más melanina, ya sea por causas endógenas o exógenas. En otras ocasiones aparecen por la acumulación de fármacos o por la inducción al aumento de producción de melanina (10 a 20\% de todos los casos de hiperpigmentación adquirida) y por depósitos focales de metales. El diagnóstico de las lesiones pigmentadas oscuras de la cavidad oral y de los tejidos periorales, en muchos casos, puede ser complicado. Por lo general, para su estudio suelen clasificarse con base en su procedencia, ya sea endógena o exógena, y a su distribución. El objetivo de este artículo consiste en realizar una revisión de las principales lesiones oscuras con implicación estomatológica, estableciendo puntos clave para el diagnóstico clínico, y proponer un algoritmo para el diagnóstico diferencial, basado en la sintomatología. Creemos que puede ser una aportación útil, ya que, si bien en la literatura consultada se han encontrado clasificaciones relativas a la procedencia de las lesiones o a su distribución, no hemos hallado ninguna que atienda a la semiología de estas lesiones.

\section{Diagnóstico diferencial de las pigmentaciones endógenas oscuras}

Las pigmentaciones endógenas tienen diversas causas, que varían dependiendo de la presencia de una sola mácula o de varias en diferentes zonas, así como de su sintomatología. La existencia de varias máculas puede indicar desde pigmentaciones fisiológicas, sin importancia patológica y en las cuales no es necesario ningún tratamiento (salvo que el paciente demande una solución estética $)^{1,2}$, hasta diversos síndromes y enfermedades en los cuales la presencia de estas pigmentaciones pueden tener una especial importancia para un diagnóstico precoz. Es el caso de la enfermedad de Addison (insuficiencia corticosuprarrenal primaria), en la que la aparición precoz en la boca de estas pigmentaciones puede anticipar el diagnóstico de esta entidad, incluso antes de que se presenten el resto de los síntomas ${ }^{3}$. Las pigmentaciones solitarias corresponden a: mácula melanótica oral, nevus melanocítico, nevus azul, melanoacantoma o lesiones graves, como el melanoma maligno. Este puede aparecer también como melanoma amelánico sin pigmentación ${ }^{2}$. En ocasiones las pigmentaciones pueden deberse a enfermedades inflamatorias de larga duración en la mucosa, como enfermedad liquenoide, pénfigo o penfigoide orales ${ }^{4}$. En estos casos la patogénesis de la pigmentación postinflamatoria no está muy clara y puede observarse con mayor frecuencia en individuos de piel oscura ${ }^{1}$. En las tablas 1 y 2 se resume la información más relevante relativa a las pigmentaciones endógenas, según nuestra propuesta de clasificación.

\section{Diagnóstico diferencial de las pigmentaciones} exógenas oscuras

Las pigmentaciones exógenas pueden deberse a la acción de determinados compuestos que estimulan a los melanocitos a

\footnotetext{
* Autor para correspondencia.

Correo electrónico: evalopez1973@live.com (E. López-Montoro). https://doi.org/10.1016/j.piel.2017.12.005

0213-9251/(C) 2018 Elsevier España, S.L.U. Todos los derechos reservados.
} 
Tabla 1 - Pigmentaciones orofaciales endógenas únicas y múltiples sin sintomatología asociada

\begin{tabular}{|c|c|c|c|}
\hline Entidad & Clínica y localización más frecuente & $\begin{array}{l}\text { Pruebas } \\
\text { diagnósticas }\end{array}$ & Diagnóstico diferencial \\
\hline Mácula melanótica & $\begin{array}{l}\text { Mácula pequeña, circunscrita, de marrón } \\
\text { a negro, > labio inferior, encía zona } \\
\text { anterior maxilar }{ }^{1,2}\end{array}$ & Biopsia $^{1}$ & $\begin{array}{l}\text { Melanosis fisiológica }^{1} \\
\text { Melanoma maligno }^{17}\end{array}$ \\
\hline Nevus azul & $\begin{array}{l}\text { Mácula/pápula asintomática, azul pizarra } \\
\text { o negro azulada, brillante, bordes } \\
\text { regulares, }<6 \mathrm{~mm} \text { de diámetro, piel, } \\
>\text { mucosa, }>\text { mujer, rara vez recurren, } \\
>\text { inocuos }^{1}\end{array}$ & Clínica $^{1}$ & Melanoma maligno $^{1}$ \\
\hline Melanoma maligno & $\begin{array}{l}\text { > Paladar duro }(40 \%) \text { y blando, encía } \\
\text { maxilar, > hombre, aprox. } 55 \text { años, } \\
\text { consistencia dura, marrón/negro }\end{array}$ & Biopsia $^{1}$, & $\begin{array}{l}\text { inmunohistoquímica }{ }^{18} \text {, } \\
\text { exploración clínica }^{17}\end{array}$ \\
\hline \multicolumn{4}{|l|}{$\begin{array}{l}\text { Melanoma, mácula } \\
\text { melanótica, nevo } \\
\text { pigmentado oral, melanosis } \\
\text { fumador, tatuaje amalgama } \\
\mathrm{Ag} \text {, sarcoma de Kaposi }{ }^{17}\end{array}$} \\
\hline Nevus melanocítico & $\begin{array}{l}\text { Mácula/pápulas redondas/ovales } \\
\text { circunscritas, marrón-azul o negro, > piel } \\
\text { clara, > mucosa (labios/encía/paladar/ } \\
\text { mucosa) }\end{array}$ & $\begin{array}{l}\text { Biopsia }^{1} \\
\text { dermoscopia }^{19}\end{array}$ & Melanoma maligno 20 \\
\hline Melanoacantoma & $\begin{array}{l}>\text { Raza negra, jóvenes, máculas }^{16}, \\
>\text { mucosa bucal, encías, paladar, }^{2} \\
\text { labios } \\
\text { rápidamente, oparición repentina, crece } \\
\text { múltiple }{ }^{16,20}\end{array}$ & Biopsia $^{16}$ & $\begin{array}{l}\text { Melanoma }{ }^{16,20} \text {, mácula racial/ } \\
\text { melanótica oral, petequias, } \\
\text { tatuaje amalgama, fármacos, } \\
\text { postinflamatorio, genética }^{20}\end{array}$ \\
\hline Melanosis fisiológica & $\begin{array}{l}\text { Encía adherida, pigmentaciones múltiples } \\
\text { color marrón claro a casi negro, difusas } \\
\text { y bilaterales }\end{array}$ & Clínica $^{1}$ & Melanosis fumador ${ }^{20}$ \\
\hline Efélides & $\begin{array}{l}<1 \mathrm{~cm} \text {, uniformes y regulares, rojas o } \\
\text { marrones oscuras, }>\text { piel clara, múltiples, } \\
>\text { sol, }>\text { borde bermellón labios o tejidos } \\
\text { periorales }^{2}\end{array}$ & & \\
\hline Lentigo & $\begin{array}{l}\text { Pigmentación ligera marrón oscuro, } \\
\text { circunscrita, únicas o múltiples(asociado } \\
\text { a síndromes raros) }{ }^{2}\end{array}$ & & \\
\hline Melasma & $\begin{array}{l}\text { > En mujeres, patrón simétrico, marrón } \\
\text { a pardo grisáceo, hiperpigmentación } \\
\text { múltiple de rostro zona malar y } \\
\text { centrofacial }^{21}\end{array}$ & $\begin{array}{l}\text { Clínica, lámpara } \\
\text { de Wood }{ }^{21}\end{array}$ & Lupus eritematoso discoide ${ }^{21}$ \\
\hline Síndrome de Laugier-Hunziker & $\begin{array}{l}\text { Máculas ovales/redondas, marrón claro } \\
\text { a azul grisáceo y negro, > labio inferior, } \\
\text { mucosa oral, paladar blando/duro y } \\
\text { encías, palmas manos y pies }{ }^{1} \text {, } \\
\text { melanoniquia longitudinal, > raza blanca } \\
\text { y mujer }{ }^{22}\end{array}$ & Clínica $^{22}$ & $\begin{array}{l}\text { Melanoma o síndrome de Peutz- } \\
\text { Jeghers, enfermedad de Addison, } \\
\text { liquen plano, pigmentación } \\
\text { farmacológica, racial, melanoma }_{\text {maligno }^{22}}\end{array}$ \\
\hline
\end{tabular}

melanina-fármaco o la absorción del fármaco por macrófagos, usualmente dérmicos, que lo transforman en partículas visibles bajo la influencia de la luz solar. El tratamiento a menudo se limita a evitar el sol o a interrumpir la toma del fármaco causante ${ }^{5}$. Sin embargo, existen pacientes predispuestos, a quienes los fármacos les pueden causar una reacción no específica intraoral y posteriormente inducir una hiperpigmentación postinflamatoria ${ }^{1}$. Muchos fármacos están relacionados con la presencia de máculas exógenas. Mayoritariamente son antineoplásicos, como busulfán ${ }^{2,6}$, doxorrubicina $^{2,6-8}$, ciclofosfamida ${ }^{2,6}$, carmustina (BCNU) tópica ${ }^{7}$, bleomicina ${ }^{6-8}$, daunorrubicina ${ }^{7}$, mecloretamina ${ }^{7}$, dactinomicina $^{8}$, 5-fluorouracilo ${ }^{6-8}$, metotrexato ${ }^{7,8}$, mesilato de imatinib $^{7}$, que en general producen hiperpigmentación de la 
Tabla 2 - Pigmentaciones orofaciales endógenas únicas y múltiples con sintomatología asociada

\begin{tabular}{|c|c|c|c|}
\hline Entidad & Clínica y localización más frecuente & Pruebas diagnósticas & Diagnóstico diferencial \\
\hline Melanoma maligno & $\begin{array}{l}\text { > Paladar duro (40\%) y blando, encía } \\
\text { maxilar, > hombre, aprox. } 55 \text { años, } \\
\text { consistencia dura, marrón/negro, } \\
\text { asintomático, crecimiento horizontal } \\
\text { lento, bordes asimétricos e irregulares, o } \\
\text { vertical rápido, crecimiento con } \\
\text { ulceración, sangrado, dolor y destrucción } \\
\text { ósea }^{1,18}\end{array}$ & $\begin{array}{l}\text { Biopsia }^{1}, \\
\text { inmunohistoquímica }^{18} \\
\text { exploración clínica }\end{array}$ & $\begin{array}{l}\text { Melanoma, mácula } \\
\text { melanótica, nevo pigmentado } \\
\text { oral, melanosis fumador, } \\
\text { tatuaje amalgama y sarcoma } \\
\text { de Kaposi }{ }^{17}\end{array}$ \\
\hline Hemocromatosis & $\begin{array}{l}\text { Inicia astenia, somnolencia y artralgias, } \\
\text { después dolor abdominal, pérdida libido, } \\
\text { impotencia, artropatías, pigmentación } \\
\text { gris azulada mucocutánea (paladar duro, } \\
\text { encía y mucosa bucal) y dental, depósitos } \\
\text { de ferritina y hemosiderina }{ }^{2,23}\end{array}$ & $\begin{array}{l}\text { Clínica, estudios genéticos, } \\
\text { bioquímica }^{23}, \text { biopsia } \\
\text { hepática }^{24}\end{array}$ & $\begin{array}{l}\text { Síndrome } \\
\text { pseudofibromiálgico }^{24}\end{array}$ \\
\hline Enfermedad de Addison & $\begin{array}{l}\text { Hiperpigmentación difusa, > tejido } \\
\text { cicatricial }^{25} \text {, múltiples sitios y cavidad } \\
\text { oral en general: lengua, paladar duro, } \text { mucosas }^{1,26} \text {, fatiga, debilidad, pérdida de } \\
\text { peso }^{3}\end{array}$ & $\begin{array}{l}\text { Clínica }^{2-4} \text {, prueba rápida } \\
\text { inyección de } \text { ACTH }^{26}\end{array}$ & $\begin{array}{l}\text { Síndrome de Peutz-Jeghers, } \\
\text { síndrome de Laugier- } \\
\text { Hunziker }^{22}\end{array}$ \\
\hline $\begin{array}{l}\text { Enfermedad de Von } \\
\text { Recklinghausen }\end{array}$ & $\begin{array}{l}\text { Neurofibromas múltiples, pigmentación } \\
\text { macular en piel y mucosas, color café con } \\
\text { leche }(>6) \text {, nódulos de Lisch, } \\
>\text { neurofibrosarcomas malignos }^{27}\end{array}$ & $\begin{array}{l}\text { TAC, resonancia } \\
\text { magnética }^{27}\end{array}$ & $\begin{array}{l}\text { Schwannomatosis, síndrome } \\
\text { de Watson }{ }^{27}\end{array}$ \\
\hline $\begin{array}{l}\text { Síndrome de Peutz- } \\
\text { Jeghers }\end{array}$ & $\begin{array}{l}\text { Poliposis intestinal, > riesgo carcinomas } \\
\text { gastrointestinal, páncreas, mama y } \\
\text { tiroides }^{28} . \text { Máculas marrones a } \\
\text { negras }<1 \mathrm{~mm} \text { periorales, labio inferior, } \\
\text { perinasales, conjuntivales y/o rectales }{ }^{1,2}\end{array}$ & $\begin{array}{l}\text { Clínica, hemograma, sangre } \\
\text { oculta en heces, } \\
\text { endoscopia }\end{array}$ & \\
\hline $\begin{array}{l}\text { Sarcoma de Kaposi (HHV- } \\
8 \text {, VSK) }\end{array}$ & $\begin{array}{l}\text { Máculas marrones/violáceas, parches, } \\
\text { placas y nódulos. En tronco, cara, } \\
\text { miembros y cavidad oral, > encía, paladar } \\
\text { duro, orofaringe, mucosa alveolar y dorso } \\
\text { de la lengua. El } 71 \% \text { asociado a sida }{ }^{29}, \\
\text { afectación sistémica gastrointestinal } 40 \% \\
\text { y pulmonar }\end{array}$ & $\begin{array}{l}\text { Biopsia de las lesiones } \\
\text { cutáneas }^{29}\end{array}$ & $\begin{array}{l}\text { Angiomatosis bacilar, } \\
\text { linfoma no Hodgkin e } \\
\text { infecciones cutáneas por } \\
\text { hongos o bacterias }^{29}\end{array}$ \\
\hline $\begin{array}{l}\text { Hiperpigmentación } \\
\text { postinflamatoria }\end{array}$ & $\begin{array}{l}\text { Historia o presencia de inflamación } \\
\text { y }>\text { áreas pigmentadas pardo-negro } \\
\text { adyacentes a lesiones reticulares, } \\
\text { erosivas/descamativas o vesiculares }^{10} \text {, } \\
>\text { pacientes de piel oscura }\end{array}$ & Clínica, biopsia ${ }^{10}$ & $\begin{array}{l}\text { Enfermedad liquenoide }{ }^{15} \text {, } \\
\text { pénfigo, penfigoide }{ }^{1} \text {, lupus } \\
\text { eritematoso discoide, } \\
\text { dermatitis seborreica/ } \\
\text { atópica, sarcoidosis, psoriasis } \\
\text { y otros }{ }^{10}\end{array}$ \\
\hline
\end{tabular}

piel y melanoniquia estriada, agravándose si se combina con radioterapia y en zonas fotoexpuestas ${ }^{8}$. Los antirretrovirales también están frecuentemente implicados en las pigmentaciones exógenas cutáneas, sobre todo en la zona palmoplantar, mucosa y uñas, produciendo melanoniquia estriada, de coloración parduzca. Entre ellos encontramos zidovu$\operatorname{dina}^{1,2,5,7}$, emtricitabina, indinavir y tenofovir ${ }^{7}$. Se ha encontrado relación entre hiperpigmentación de coloración marrónamarilla e incluso negro azulada en la piel y a nivel oral en el paladar duro con la toma de antimaláricos como las aminoquinolonas ${ }^{6,7,9}$. Otros fármacos, como amiodarona ${ }^{7}$, minociclina $^{6,7}$, premarin ${ }^{2,6,7,10}$, acetaminofeno ${ }^{11}$, metoxaleno $^{12}$, ketoconazol ${ }^{2,6}$ y clofazamina ${ }^{1}$, se han relacionado con la aparición de hiperpigmentación en pacientes expuestos a los mismos.

En el caso de la acumulación tóxica por metales, en la mayoría de los casos el diagnóstico se puede realizar solamente si se lleva a cabo un estudio muy detallado de los hábitos alimentarios, el uso de suplementos dietéticos, los medicamentos que toma, el estilo de vida, la profesión, el lugar de residencia o las exposiciones ambientales del paciente expuesto.

La toxicidad de los metales pesados puede afectar a cualquier sistema de órganos, como el sistema gastrointestinal y el hematopoyético, así como a la función cardiovascular y renal. Depende de muchos factores: el tipo de elemento, su forma química, las dosis administradas, la duración de la exposición y, sobre todo, la edad del paciente, siendo más grave en niños.

Para el diagnóstico de las lesiones pigmentarias generalizadas, son útiles las pruebas básicas de laboratorio (citología, hemograma, análisis de orina, función hepática y renal, electrólitos), el diagnóstico por imagen, la capilaroscopia y la histopatología ${ }^{13}$. Ante lesiones únicas en que se sospeche acumulación de metal, es útil la realización de radiografías intraorales ${ }^{1}$.

La absorción sistémica de metales conduce a un depósito generalizado, mientras que la exposición traumática y la 
Tabla 3 - Lesiones orofaciales pigmentadas exógenas únicas y múltiples sin sintomatología asociada

\begin{tabular}{|c|c|c|c|}
\hline Entidad & Clínica y localización más frecuente & Pruebas diagnósticas & Diagnóstico diferencial \\
\hline $\begin{array}{l}\text { Melanosis } \\
\text { fumador }\end{array}$ & $\begin{array}{l}\text { Máculas marrón, gris o negro, > mucosa oral, encía } \\
\text { inferoanterior }{ }^{30} \text {, asintomáticas, } 25-31 \% \\
\text { fumadores } \text { fum }^{2,31} \text {, en meses/años tras deshabituación } \\
\text { suele remitir hiperpigmentación }\end{array}$ & $\begin{array}{l}\text { Clínica y antecedentes } \\
\text { tabáquicos }\end{array}$ & $\begin{array}{l}\text { Melanosis fisiológica y } \\
\text { máculas melanóticas }\end{array}$ \\
\hline $\begin{array}{l}\text { Melanosis por } \\
\text { fármacos }\end{array}$ & $\begin{array}{l}\text { Máculas gris negruzcas, difusas, generalmente } \\
\text { asintomáticas }{ }^{7} \text {. Dependiendo del fármaco, } \\
\text { hiperpigmentación de la piel en general, zona } \\
\text { palmoplantar, mucosa oral }{ }^{2,6} \text {, melanoniquia } \\
\text { estriada }^{7} \text { o con sintomatología (eritema acral) }\end{array}$ & Clínica, retirada del fármaco ${ }^{5}$ & \\
\hline $\begin{array}{l}\text { Tatuaje por } \\
\text { amalgama }\end{array}$ & $\begin{array}{l}\text { Mucosa lacerada. Mácula asintomática gris-azul, } \\
<1 \mathrm{~cm} \text {, tatuaje }>\text { único o múltiple. El 50\% mucosa } \\
\text { alveolar, encía, } 20 \% \text { mucosa bucal }{ }^{1,2,33}\end{array}$ & Evaluación radiográfica ${ }^{1,2}$ & Melanoma $^{1}$ \\
\hline $\begin{array}{l}\text { Argiria } \\
\text { localizada }\end{array}$ & $\begin{array}{l}\text { Asintomática, acumulación en paladar, } \\
\text { pigmentación cartílago oreja, uñas, mucosas }{ }^{21}\end{array}$ & $\begin{array}{l}\text { Histopatología o historia } \\
\text { positiva de la ingesta de } \\
\text { plata }^{21,34}\end{array}$ & $\begin{array}{l}\text { Melanoma }{ }^{21} \text {, tatuaje, } \\
\text { nevus azul, lesiones } \\
\text { melanocíticas }\end{array}$ \\
\hline
\end{tabular}

Tabla 4 - Pigmentaciones orofaciales exógenas únicas y múltiples con sintomatología asociada

\begin{tabular}{|c|c|c|c|}
\hline Entidad & Clínica y localización más frecuente & Pruebas diagnósticas & Diagnóstico diferencial \\
\hline $\begin{array}{l}\text { Dermatitis de } \\
\text { contacto } \\
\text { fototóxica }\end{array}$ & $\begin{array}{l}\text { Picazón o ardor con eritema e } \\
\text { hiperpigmentación en el lugar de } \\
\text { aplicación, > tez oscura }{ }^{35}\end{array}$ & $\begin{array}{l}\text { Prueba con parche, historia clínica } \\
\text { detallada }^{35}\end{array}$ & \\
\hline Exantema fijo & medicamentoso & $\begin{array}{l}\text { Placas recurrentes en ubicaciones fijas, } \\
>\text { labios, genitales y zonas acras tras cada } \\
\text { exposición al fármaco (> antibiótico y } \\
\text { AINE), multiformes, delimitadas, } \\
\text { redondas/ovaladas de eritema y edema, } \\
\text { color violáceo oscuro a marrón, a veces } \\
\text { vesícula o ampolla, normalmente no } \\
\text { pruriginoso }^{36}\end{array}$ & $\begin{array}{l}\text { Clínica: > } 30 \mathrm{~min} / \text { día tras } \\
\text { exposición al fármaco }{ }^{37} . \text { Se } \\
\text { resuelve con hiperpigmentación } \\
\text { postinflamatoria }^{36}\end{array}$ \\
\hline $\begin{array}{l}\text { Melanosis por } \\
\text { fármacos }\end{array}$ & antineoplásicos & $\begin{array}{l}\text { Máculas gris pardo, piel palmoplantar, } \\
\text { mucosa oral. Melanoniquia estriada, } \\
\text { eritema acral en algunos } \operatorname{casos}^{32}\end{array}$ & \\
\hline $\begin{array}{l}\text { Melanosis por } \\
\text { metales }\end{array}$ & $\begin{array}{l}\text { Pigmentaciones en la piel en general y } \\
\text { zona esclerótica a nivel oral en paladar, } \\
\text { en encía marginal. A nivel sistémico } \\
\text { encontraremos malestar general, } \\
\text { neuropatía periférica, vómitos, } \\
\text { diarrea }^{34,13}\end{array}$ & $\begin{array}{l}\text { Histopatología }{ }^{21,34} \text {. Clínica y analítica } \\
\text { general }^{13}\end{array}$ & $\begin{array}{l}\text { Melanoma }{ }^{21} \text {, enfermedad de } \\
\text { Addison, hemocromatosis, } \\
\text { hiperpigmentación inducida por } \\
\text { fármacos }^{34}\end{array}$ \\
\hline
\end{tabular}

aplicación tópica dan como resultado un área localizada de acumulación. La deposición de metales presenta clínicamente una hiperpigmentación, que suele ser irreversible. Algunos depósitos son fotoacentuados, lo que empeora el cuadro clínico desde el punto de vista estético ${ }^{14}$.

En las tablas 3 y 4 se resume la información más relevante relativa a las pigmentaciones exógenas, según nuestra propuesta de clasificación.

\section{Discusión}

La descripción de las lesiones pigmentadas oscuras de este trabajo, si bien no es exhaustiva, ofrece una visión general de la amplitud del tema.
La hiperpigmentación parece ser una forma de reactividad del organismo frente a diversas noxas, entre las cuales se incluyen: farmacoterapia, cosmética, tabaco, síndromes o enfermedades sistémicas, entre otras. Entidades inflamatorias como la enfermedad liquenoide, el pénfigo o penfigoide orales, así como la enfermedad periodontal crónica, a veces se asocian con deposición de melanina en el tejido conectivo, resultando en un oscurecimiento de la zona mucosa ${ }^{1}$.

En odontología y en medicina conviene consensuar criterios diagnósticos clínicos e histopatológicos precisos para distinguir y diferenciar las lesiones pigmentadas. Ello permite evaluar un eventual potencial de malignización, así como elegir el tratamiento más adecuado. En cada caso, deberá realizarse un análisis sistemático, que incluya la historia clínica pormenorizada y un examen completo del 


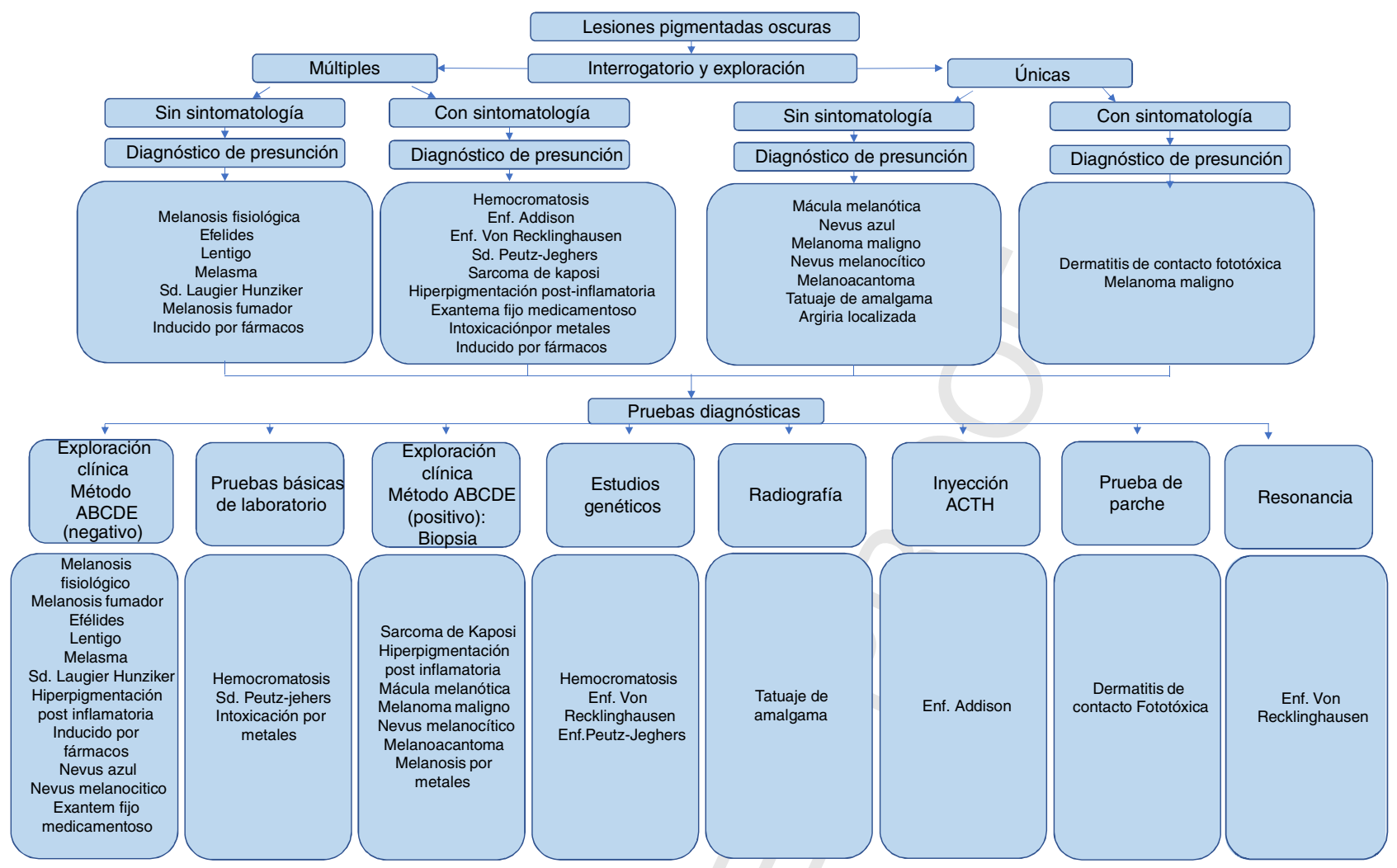

Fig. 1 - Algoritmo para el diagnóstico diferencial de las melanosis orofaciales y pruebas complementarias en función del diagnóstico de presunción.

paciente, con el fin de establecer un diagnóstico diferencial razonable.

Muchas lesiones pigmentadas pueden diagnosticarse clínicamente con base en el tamaño, la forma, el color y la distribución, contrastadas con otros datos de la historia clínica. En los casos en los que las características clínicas sean atípicas o sospechosas de malignidad, se deberá realizar una biopsia, para ayudar a establecer el diagnóstico. Ocasionalmente, pueden requerirse técnicas inmunohistoquímicas $\mathrm{u}$ otras pruebas complementarias ${ }^{1}$. Es particularmente importante para descartar el melanoma, ya que puede iniciarse como una simple hiperpigmentación de la mucosa.

Aunque parece haber una relación entre la aparición de hiperpigmentación y el uso de determinados fármacos, la evidencia científica es deficiente, ya que la mayoría de los artículos consultados sobre la relación entre el uso de un fármaco y la hiperpigmentación se basan en pacientes individuales (nivel de evidencia 3) y no se ha encontrado una relación causal clara ${ }^{6}$.

En prácticamente todos los artículos consultados relativos a la etiología de las máculas producidas por fármacos se emplean términos como "posiblemente» y "probablemente», lo que indica que el mecanismo de acción no está claro, por lo que son necesarios más estudios para aclarar cómo actúan los fármacos en la aparición de las máculas. Ante la sospecha de melanosis oral inducida por fármacos, debe tenerse en cuenta que la cavidad oral puede no ser el único sitio involucrado. Por este motivo deben explorarse otras zonas del cuerpo, en busca de más lesiones, así como comprobar si la supresión o el cambio de fármaco las hace desaparecer ${ }^{5,15}$.

El diagnóstico diferencial de las lesiones solitarias debe realizarse entre melanosis exógenas por metal, iatrogenia, melanosis cutáneas benignas o lesiones malignas. El melanoma maligno suele ser una lesión solitaria de consistencia dura $^{16}$, que, en la mayoría de los casos, se encuentra en el paladar o la encía, requiriendo la realización de una biopsia ${ }^{1,2}$. En cambio, la distribución multifocal o difusa de la pigmentación apunta, en general, a una causa sistémica, la toxicidad de un fármaco, relación con el consumo de tabaco o una melanosis fisiológica.

Para concluir, y habida cuenta de que en la literatura consultada no se han hallado clasificaciones basadas en la semiología, proponemos su clasificación con base en pigmentaciones exógenas, endógenas, asintomáticas, sintomáticas, únicas o múltiples. Si bien existen muchas sustancias químicas implicadas en la melanosis oral, a veces la relación causal no está del todo clara, aun cuando se ha comprobado la relación entre la frecuencia de la administración de dichos fármacos y la aparición de las máculas. Para determinar la causa de la hiperpigmentación son imprescindibles una buena anamnesis y una exploración clínica que incluya la observación de las estructuras orales y extraorales (color, distribución, signos/síntomas). En respuesta a nuestro objetivo, aportamos un algoritmo que ayuda al diagnóstico diferencial de las lesiones pigmentarias (fig. 1). 


\section{Puntos clave}

- Las pigmentaciones orofaciales generalmente se forman por la inducción de los melanocitos a producir más melanina, ya sea por causas endógenas o exógenas.

- Las máculas oscuras orales en muchas ocasiones aparecen por la acumulación de fármacos y/o la inducción de los mismos al aumento de producción de melanina y por depósitos focales de metales por acumulación tóxica.

- En odontología y en medicina conviene consensuar criterios diagnósticos clínicos precisos para distinguir y diferenciar las lesiones pigmentadas, lo que permitirá evaluar el riesgo de malignización y elegir el tratamiento más adecuado.

- Deberá realizarse un análisis sistemático, que incluya la historia clínica pormenorizada y un examen completo del paciente, con el fin de alcanzar un diagnóstico diferencial razonable.

- Disponer de una clasificación de las melanosis orofaciales con base en su procedencia exógena o endógena, distribución y sintomatología ayudará a establecer un diagnóstico de presunción que contribuirá a elegir las pruebas complementarias necesarias para alcanzar al diagnóstico de certeza.
1. Gondak RO, da Silva-Jorge R, Jorge J, Lopes MA, Vargas PA. Oral pigmented lesions: Clinicopathologic features and review of the literature. Med Oral Patol Oral Cir Bucal. 2012;17:919-24.

2. Meleti M, Vescovi P, Mooi WJ, van der Waal I. Pigmented lesions of the oral mucosa and perioral tissues: A flow-chart for the diagnosis and some recommendations for the management. Oral Surg Oral Med Oral Pathol Oral Radiol Endod. 2008;105:606-16.

3. Alemparte Pardavila E, Martínez Melgar JL, Piñeiro Sande N, Castellano Canda P, Rodríguez García JC. Enfermedad de Addison: el reto de un diagnóstico precoz. An Med Interna. 2005;22:332-4.

4. Chimenos-Küstner E, Larred-Conde N, Marques-Soares MS, López-López J. Manifestaciones orales de toxicidad sistémica: patología liquenoide. Piel. 2015;30:644-9.

5. Lee A, Thomson J. Drug-induced skin reactions. En: Lee A, editor. Adverse drugs reactions. 2 nd ed. London, UK: Pharmaceutical Press; 2006. p. 125-52.

6. Krause W. Drug-induced hyperpigmentation: A systematic review. J Dtsch Dermatol Ges. 2013;11:644-51.

7. Capelli L, Garuti RA, Carmona Cuello LE, Sanz A, Renata Oborski M, León SA, et al. Hiperpigmentación cutáneomucosa y ungular secundaria a tenofovir. Arch Argent Derm. 2013;63:23-6.

8. Guillot B, Bessis D, Dereure O. Mucocutaneous side effects of antineoplastic chemotherapy. Expert Opin Drug Saf. 2004;3:579-87.

9. Kleinegger CL, Hammond HL, Finkelstein MW. Oral mucosal hyperpigmentation secondary to antimalarial drug therapy.
Oral Surg Oral Med Oral Pathol Oral Radiol Endod. 2000;90:189-94.

10. Vashi NA, Kundu RV. Facial hyperpigmentation: Causes and treatment. Br J Dermatol. 2013;169 Suppl 3:41-56.

11. Pineda R, Rodriguez E, Nuñez T, Martinez P, Pelta R, Barrio R. Non-immediate skin reactions to paracetamol. A report of two cases. Allergy. 2012;67:307-451.

12. Romero Pareyón LA. Dermatosis reaccionales. Rev Fac Med UNAM. 2003;46:148-51.

13. Ferrer A. Metal poisoning. An Sist Sanit Navar. 2003;26: $141-53$.

14. Greenberg JE, Lynn M, Kirsner RS, Elgart GW, Hanly AJ. Mucocutaneous pigmented macule as a result of zinc deposition. J Cutan Pathol. 2002;29:613-5.

15. Chimenos-Küstner E, Larred-Conde N, Marques-Saores MS, López López J. Oral manifestations of systemic toxicity: Lichenoid diseases. Piel. 2015;30:644-9.

16. Carlos-Bregni R, Contreras E, Netto AC, Mosqueda-Taylor A, Vargas PA, Jorge J, et al. Oral melanoacanthoma and oral melanotic macule: A report of 8 cases, review of the literature, and immunohistochemical analysis. Med Oral Patol Oral Cir Bucal. 2007;12:374-9.

17. Mohan M, Sukhadia VY, Pai D, Bhat S. Oral malignant melanoma: Systematic review of literature and report of two cases [Internet]. Oral Surg Oral Med Oral Pathol Oral Radiol. 2013;116:e247-54.

18. González García R, Naval Gías L, Martos PL, Hyun Nam-Cha S, Rodríguez Campo FJ, Muñoz Guerra MF, et al. Melanoma de la mucosa oral. Casos clínicos y revisión de la literatura/Melanoma of the oral mucosa. Clinical cases and review of the literature. Med Oral Patol Oral Cir Bucal. 2005;10:264-71.

19. Kaminska-Winciorek G, Spiewak R. Tips and tricks in the dermoscopy of pigmented lesions. BMC Dermatol. 2012;12:14.

20. Caviglia S. Melanoacantosis bucal: diagnóstico y tratamiento de un caso clínico. Rev ADM. 2014;71:28-30.

21. Patel A, Kubba R, Kubba A. Clinicopathological correlation of acquired hyperpigmentay disorders. Indian J Dermatol Venereol Leprol. 2013;79:367-75.

22. Vilar Coromina N, Ferrándiz L, Moreno-Ramírez D, Camacho Martínez F. Síndrome de Laugier-Hunziker. Piel. 2009;24:304-6.

23. Schemel-Suárez M, López-López J, Chimenos-Küstner E. Dental pigmentation and hemochromatosis: A case report. Quintessence Int (Berl). 2017;48:155-9.

24. Sánchez Ayala JJ, Marne Santamaría D, Marcaide Ruiz de Apodaka MA. Hipótesis profesional de la hemocromatosis. Med Segur Trab. 2010;56:165-85.

25. Patel LM, Lambert PJ, Gagna CE, Maghari A, Lambert WC. Cutaneous signs of systemic disease. Clin Dermatol. 2011;29:511-22.

26. Eisen D. Disorders of pigmentation in the oral cavity. Clin Dermatol. 2000;18:579-87.

27. Evans MG, Poulsen RR, Mardones SC. Enfermedad de Von Recklinghausen y embarazo. Rev Chil Obstet Ginecol. 2002;67:153-6.

28. Ferreiro CM, Harris DP, Larraín BF, Duarte GI, Repetto LG. Una familia con síndrome de Peutz-Jeghers. Rev Chil Pediatr. 2000;71:214-9.

29. Govindan B. Recapitulation of acquired immuno deficiency syndrome associated Kaposi's sarcoma. Indian J Sex Transm Dis. 2016;37:115-22.

30. Kalampalikis A, Goetze S, Elsner P. Isolated hyperpigmentation of the oral mucosa due to hydroxychloroquine. J Dtsch Dermatol Ges. 2012;10:921-2.

31. Villarroel-Dorrego M, López-Labady J, Sánchez Traslaviña N, Orfali Azrak C, Prieto Castro K, Sandoval Márquez A. Melanosis bucal asociada a tabaquismo inhalado en un 
Q4 grupo de venezolanos: estudio clínico transversal. Acta Odontol Venez. 2013;51:2.

32. Allevato M. Efectos adversos cutáneos de la terapia antineoplásica. Act Terap Dermatol. 2008;31:78-91.

33. Boyd AS, Seger D, Vannucci S, Langley M, Abraham JL, King LE. Mercury exposure and cutaneous disease. J Am Acad Dermatol. 2000;43:81-90.

34. Kubba A, Kubba R, Batrani M, Pal T. Argyria an unrecognized cause of cutaneous pigmentation in Indian patients: A case series and review of the literature. Indian J Dermatol

Q5 Venereol Leprol. 2013;79:805-11.

35. Mehta SS, Reddy BS. Cosmetic dermatitis - Current perspectives. Int J Dermatol. 2003;42:533-42.

36. Pai VV, Bhandari P, Kikkeri NN, Athanikar SB, Sori T. Fixed drug eruption to fluconazole: A case report and review of literature. Indian J Pharmacol. 2012;44:643-5.

37. Bjekic M, Markovic M, Sipetic S. Fixed drug eruption caused by tadalafil-Case report. An Bras Dermatol. 2013;88:617-9.

Cómo citar este artículo: López-Montoro E, Chimenos-Küstner E, Diagnóstico diferencial de las melanosis orofaciales. Piel (Barc). 2018. https:// doi.org/10.1016/j.piel.2017.12.005 